\title{
Self-check Home Exercises Improves Static and Dynamic Balance, Fall Efficacy, and Quality of Life in Patients with Parkinson`s Disease
}

\author{
Jin-hong Kim ${ }^{1}$, Jun-hyuck Park ${ }^{1}$ and Jong-eun Yim ${ }^{2 *}$ \\ ${ }^{1}$ Department of Physical Therapy, The Graduate School of Sahmyook Universi- \\ ty, Seoul, Korea \\ ${ }^{2}$ Department of Physical Therapy, Sahmyook University, Seoul, Korea \\ *jeyim@syu.ac.kr
}

\begin{abstract}
Parkinson's disease is a chronic progressive disease of the nervous system associated with the basal ganglia and is characterized by rigidity, bradykinesia, tremor, and instability of posture. In addition, it causes indirect disabilities and complications. The aim of this study was to evaluate the effects of self-check home exercise on fall prevention in patient with Parkinson's disease. The present study included 20 patients who met the study criterion, which was a diagnosis of Parkinson's disease. The present study was conducted over 12 weeks in the exercise therapy center of the G University Hospital. Periodically, once a month at the first, fifth, and ninth-week sessions, a neurosurgeon, a rehabilitation physician, a nurse in charge of neurosurgery programs dedicated to Parkinson's disease, a physiotherapist, and an occupational therapist attended the home exercises program to provide group lessons to subjects, to provide feedback regarding the home exercises program, and to carry out a self-monitoring checklist performance rate. The results showed significant differences in TUG, FRTA, FRTP, FES, STS, 10MWT, MBI, and QoL ( $p<.05)$. The present study involved showing patients with Parkinson's disease how to easily perform home exercises and how to provide ongoing feedback for motivation. The home exercises program of the present study is expected to improve the lives of patients with Parkinson's disease.
\end{abstract}

Keywords: Parkinson's disease, self-check, home exercise

\section{Introduction}

The development of medical technology, growth of the economy, and improvement of living standards have brought about an increase in the elderly population in Korea. This increase has created social problems, population aging, etc. According to statistics released by the Korean Statistical Information Service in 2013, the elderly population aged over 65 years, accounts for $7.33 \%$ of the total population. Thus, Korea is expected to become an aged society (14\%) in 2018 and a super-aged society (20\%) in 2026. As Korea becomes a rapidly aging society, aging-associated diseases are increasing accordingly.

A typical aging-associated disease is Parkinson's disease. A review of aging-associated disease incidences for 10 years revealed that Parkinson's disease had the second highest growth rate after dementia. Parkinson's disease was first described by James Parkinson in 1817 and is a chronic progressive disease of the nervous system associated with the basal ganglia, and is characterized by rigidity, bradykinesia, tremor, and instability of posture. In addition, it causes indirect disabilities and complications [1]. Major symptoms include

* Corresponding Author 
disturbances of movement and gait, changes in sensation, cognitive and behavioral disorders, functional disorders of the autonomic nervous systems, gastrointestinal changes, and cardiovascular changes. Disease onset is insidious and progress of the disease is slow. Inability to perform routine functions, roles, and activities often occurs in patients with Parkinson's disease [2].

Therapeutic approaches for patients with Parkinson's disease include medication, surgical operations, and exercises. For medication, levodopa is used to supplement the lack of dopamine in the basal ganglia of the brain to counteract the imbalance in neurotransmitters. Levodopa is known as the most effective anti-Parkinson drug but it has been reported to cause exercise complications such as movement agitation, dyskinesias, etc., or psychological side effects including hallucinations and delusions [3]. The side effects of the drug increase the incidence of surgical therapy. Thus, surgery therapy is preferentially applied to the patients who do not respond to drug treatment or have severe side effects. However, surgery is expensive and results in postoperative side effects, which may result in a worsened quality of life [4].

Conversely, non-pharmacologic non-invasive exercise therapy is known to promote brain-derived neurotrophic factors, prevent atrophy of the brain, and affect cognitive abilities. It is also known to promote the secretion of glial-derived neurotrophic factors, increase the uptake of dopamine, and prevent the death of the motor cell [5-7]. Due to the effects of exercise therapy, many exercise therapies targeted at patients with Parkinson's disease have been conducted. A review of the effects of exercise therapy found that the improvement of the ability to balance may have a positive impact on fall prevention. In addition, it has been reported that exercise therapy improves walking ability, activities of daily living (ADL), and enhances the quality of life in patients and their caregivers $[4,8$ 10]. Moreover, it causes functional improvement in movement and has positive effects on mental status of patients such as attention, recognition, and emotional control [11].

However, patients with Parkinson's disease experience difficulties in regularly visiting hospitals and receiving exercise therapy due to other complications, such as serious deterioration of balance, cognitive impairment, and depression, and also because of the risks of accidents, such as falls [12]. Furthermore, patients with Parkinson's disease have other problems including the burden of medical expenses due to ongoing medical visits, provision of transport to visit the hospital as an outpatient and corresponding costs, and the physical and mental burdens on the caregiver. This causes functional deterioration and worsening conditions as well as psychological problems $[13,14]$. Considering these problems, home exercise therapy has been often used to reduce the risk of falling accidents, bring about medical cost-effectiveness, and reduce costs and burdens associated with securing transportation to health facilities. Specifically, according to previous studies, home exercises, such as fall prevention programs for the elderly, balance promotion programs for stroke patients, and postural control programs for patients with Parkinson's disease, were widely applied in various diseases, and the effects were demonstrated [13, 15]. However, despite the many benefits of home exercises, factors that militate against the effects of home exercises have been pointed out, which include a low rate of exercise performance and incorrect exercise performance by patients in situations where it is difficult for the therapist to carry out sustainable management [15]. Therefore, the present study attempted to demonstrate the effects of more advanced home exercises by allowing patients to check themselves using daily self-tests and regularly training patients and their caregivers to overcome the shortcomings of more advanced home exercises. 


\section{Methods}

\subsection{Study Design}

The present study was based on a cross-sectional study design. Groups were divided into a pre-tested home exercises program applied group and a post-tested home exercises program applied group.

\subsection{Participants}

The present study included 20 patients who met the study criterion, which was a diagnosis of Parkinson's disease in the department of neurology of G University in I Metropolitan City. The patients were on anti-Parkinson medication and were all in an "onstate" status indicating that they showed drug effects when tested. All the subjects were informed about the contents of the present study and consented to the experiments. The inclusion criterion of the present study was a diagnosis of Parkinson's disease by a neurologist. Patients able to walk without a walking aid, who had atypical Parkinson's syndromes such as progressive supranuclear palsy or manifold atrophy, who had symptomatic parkinsonism due to medication, or patients with cognitive disabilities were excluded. In addition, subjects who did not attain $70 \%$ in the 12 -week performance rate of home exercises using a self-check list were excluded.

\subsection{Procedure}

The present study lasted over 12 weeks in the exercise therapy center of the G University Hospital. Periodically once a month at the first, fifth, and ninth-week sessions, a neurosurgeon, a rehabilitation physician, a nurse in charge of neurosurgery programs dedicated to Parkinson's disease, a physiotherapist, and an occupational therapist attended the home exercises program to provide group lessons to subjects, to provide feedback regarding the home exercises program, and to carry out the self-monitoring checklist performance rate. In addition, patients' caregivers were encouraged to participate in the home exercises program so that patients could perform the home exercises program being clarified about its purpose and procedure. Details were provided on how to process the home exercises program conducted by experienced physiotherapists, and occupational therapists. Moreover, fall prevention education was given. Materials containing the photos and methods of the home exercises program that subjects could refer to after returning home were distributed with a self-monitoring checklist. On the fifth week, subjects were retrained to understand the home exercises program. Fine hand skills training and ADL training were given to subjects and a performance rate inspection with a self-monitoring checklist was conducted. On the ninth week, the home exercises program was conducted and included a training of the facial muscles, voice training, and a questionnaire assessing patient satisfaction with respect to the home exercises program. For participants in the present study, pre- and post-evaluation of subjects were conducted for a one-week period before and after the research was conducted. A questionnaire-based survey was performed assessing items including balance, walking, muscle strength, falling indexes, ADL, and quality of life in patients with Parkinson's disease.

\subsection{Methods}

Subjects were asked to perform the home exercises program once a day in a relaxed condition at home, using the knowledge gained in the group lessons and the brochure provided. The program included four movements: sit and stand, wall push-ups, lying down and lifting the leg, and lying down and lifting the buttocks. A total of three sets needed to be conducted 15 times per set. A rest period of 2 minutes was allowed between 
sets. In order to prevent injuries, all the joints were stretched for about 10 minutes before the start of the home exercises program [16]. After completing the home exercises program, subjects were allowed to mark their accomplishment on a self-monitored checklist.

\subsection{Assessment Tool and Data Collection}

2.5.1. Ten-meter Walk Test: While the subject walked down a section totaling $10 \mathrm{~m}$, the time required to walk a 6-m section was measured using a second clock [17-19]. The assessment of walking speed is a measurement method with high reliability and validity [20]. The average of the values measured two times consecutively was used as the final value.

2.5.2. Timed Up and Go Test: The timed up and go (TUG) test is used to evaluate the walking and balance ability in the elderly and was developed to improve objectivity and reliability. For the test procedure, the subject is asked to stand up from a chair with armrests at the word "Start," to walk for $3 \mathrm{~m}$ at the most stable and comfortable speed, to walk back to the original location, and to sit in the chair. The time from the word "Start" to the time when the patient sits back on the chair is recorded in seconds using a stopwatch. The time was measured two times and the average value was adopted as the final value. The intra-rater and inter-rater reliability of this test was $r=.99$ and $r=.98$, respectively [21].

2.5.3. Functional Reach Test: The functional reach test (FRT) was carried out to evaluate the patient's ability to balance. Duncan et al. (1990) reported that this test has a significant relation to the dynamic balance that predicts falls in the elderly. They suggested that if the measured value is less than $15.24 \mathrm{~cm}$, the balance ability decreases which increases the risk of falling [22]. For the start stance, the subject was asked to clench the two fists in a standing position with both feet apart in shoulder-width on the fixed supporting surface, to extend the elbow, and to bend the shoulder joint forward at $90^{\circ}$. While maintaining a position horizontal to the installed bar at the height of the acromion, the subject was asked to stretch the arm to the maximum and to maintain it for 5 seconds without losing balance. In this posture, the first point of the third metacarpal distally was recorded, this point was allowed to stretch maximally and the distance between the first and the last point was measured in centimeters. The functional reach test carried back and forth. Each arm was measured two consecutive times and the average value was used. In this test, the intra-rater reliability was $r=.89$ and the interrater reliability was $r=.98[23]$.

2.5.4 Sit-to-stand Test: The sit-to-stand (STS) test is used to evaluate lower extremity strength muscle-test and measures the number of times a patient sits down and stands up from a chair with a backrest and an armrest. During the procedure, subjects were asked to cross their arms on the trunk and to minimize the movement of the arms. The evaluator then pressed the start button of the stopwatch when the "Start" command was issued to the subject and the stop button when the "Stop" command was given. 
2.5.5. Falls Efficacy Scale: The falls efficacy scale (FES) was measured using a Korean FES version of the scale developed by Tinetti et al. (1990) [24]. A person-to-person interview with patients was conducted to identify any fears in performing 10 different actions on a scale of 1 to 10. Subjects who expressed much fear of falling were assigned 10 points while those who did not express any fears of falling were assigned 0 points. The total score ranges from a minimum of 10 to a maximum of 100 points. Lower scores mean less fear of falling. The internal agreement of this scale was validated with Cronbach's $\alpha=0.92$ [25].

2.5.6. Korean-version of the Modified Barthel Index: The Korean version of the modified Barthel index (K-MBI) was developed based on independence in carrying out basic ADL. This evaluation method reflects daily living abilities, independent functions, and changes in improved functions in patients with Parkinson's disease. Ten items were evaluated including personal hygiene, bathing, eating, waste management, climbing stairs, dressing, stool control, urine control, and movement from walking/chair and car/bed. This test is a 5-point scaled test, from total dependence to independence, with a maximum score of 100. Inter-rater reliability was $0.95-0.98$ and test-retest reliability was $97-1.00$ (very high) $[26,27]$.

2.5.7 Quality of Life Index in Patients with Parkinson's Disease: Parkinson's disease questionnaire (PDQ)-39 developed by Jenkinson et al. (1995) is a self-survey method for evaluating the quality of life of patients with Parkinson's disease and consists of 8 scales and 39 questions. The 8 scales comprise the following: movements (10 items), ADL (6), emotional aspects (6), symptoms (4), social support (3), cognition (4), communication (3), and physical insecurity ( 3 items) [28]. The internal agreement of these test questions is evident with a Cronbach's $\alpha=0.59-0.94$ and an inter-rater reliability of $r=0.67-0.87$ [29]. The PDQ-8 was designed to select one most critical question from each of the 8 scales of PDQ-39. PDQ-8 and PDQ-39 have a high correlation (Pearson's $r=0.96$, ICC $=0.95$ ) [29]. The evaluation was conducted by checking 5 different answers to each of the 8 questions via a one-on-one interview involving the participant and the therapist. The contents of the answer are "Not at all" (0 points), "Yes, sometimes" (1 point), "Yes, often" ( 2 points), "Yes, very often" ( 3 points), and "Always" (4 points). Scores range from 0 points (lowest) to 32 points (highest). If calculated based on 100 points, higher points mean a higher quality of life, while lower points imply a lower quality of life.

\section{Results}

Eighteen patients with Parkinson's disease participated in the study. The general characteristics of the study subjects are shown in Table 1 .

Table 1. General Characteristics of the Participants

\begin{tabular}{lc}
\hline & $\begin{array}{c}\text { Experimental group } \\
(\mathrm{n}=18)\end{array}$ \\
\hline Sex (male/female) & $18(5 / 13)$ \\
Age (years) & $69.83 \pm 6.59$ \\
Height $(\mathrm{cm})$ & $158.38 \pm 7.72$
\end{tabular}


Weight $(\mathrm{kg}) \quad 57.38 \pm 6.76$

Duration(year) $\quad 5.44 \pm 2.57$

Values are presented as mean \pm standard deviations.

Patients with Parkinson's disease were analyzed in terms of balance, muscle strength of the lower extremity, walking, ADL, and variables related to the quality of life. TUG, used to evaluate balance ability, showed a significant difference $(\mathrm{p}<.05)$ with a decrease of $0.98 \pm 1.73$ seconds and FRTA and FRTP which were used to evaluate balance ability, showed a significant difference ( $\mathrm{p}<.05$ ) with an increase of $2.00 \pm 3.46 \mathrm{~cm}$ and $2.16 \pm$ $3.65 \mathrm{~cm}$, respectively. FES, used to evaluate the fear of falling, showed a significant difference with a decrease of $1.66 \pm 2.52(\mathrm{p}<.05)$, not strength STS assessment showed a significant difference increasing $2.88 \pm 3.23$ times $(\mathrm{p}<.05)$. FES, used to evaluate the fear of falling, showed a significant difference $(\mathrm{p}<.05)$ with a decrease of $1.66 \pm 2.52$ and STS which was used to assess the muscle strength assessment of the lower extremity, showed a significant difference $(\mathrm{p}<.05)$ with an increase of $2.88 \pm 3.23$ times. The $10-\mathrm{m}$ walk test, used to evaluate walking ability, showed a significant difference $(\mathrm{p}<.05)$ with a decrease of $0.73 \pm 0.67$ seconds. MBI which was used to assess ADL, revealed significant differences $(\mathrm{p}<.05)$ with an increase of $5.55 \pm 6.85$ points and QoL, used to assess the quality of life, also showed significant differences $(\mathrm{p}<.05)$ with a decrease of $1.44 \pm$ 1.09 points (Table 2 ).

Table 2. Changes in The Pre-test and Post-test

\begin{tabular}{lcccc}
\hline & Pre-test & Post-test & Difference (post-pre) & $t(p)$ \\
\hline TUG (sec) & $13.59 \pm 4.81$ & $12.60 \pm 4.47$ & $-0.98 \pm 1.73$ & $2.415(0.027)$ \\
FRTA (cm) & $17.04 \pm 6.39$ & $19.04 \pm 7.02$ & $2.00 \pm 3.46$ & $2.446(0.026)$ \\
FRTP (cm) & $9.15 \pm 6.05$ & $11.31 \pm 7.18$ & $2.16 \pm 3.65$ & $2.512(0.022)$ \\
FES (score) & $12.94 \pm 7.53$ & $11.27 \pm 6.61$ & $-1.66 \pm 2.52$ & $2.805(0.012)$ \\
STS (number) & $24.61 \pm 7.22$ & $27.50 \pm 6.92$ & $2.88 \pm 3.23$ & $3.790(0.001)$ \\
10MWT (sec) & $6.57 \pm 2.30$ & $5.84 \pm 2.04$ & $-0.73 \pm 0.67$ & $4.629(0.000)$ \\
MBI (score) & $78.38 \pm 17.26$ & $83.94 \pm 14.18$ & $5.55 \pm 6.85$ & $3.439(0.003)$ \\
QoL (score) & $7.38 \pm 6.39$ & $5.94 \pm 6.59$ & $-1.44 \pm 1.09$ & $5.588(0.000)$ \\
\hline
\end{tabular}

Values are presented as mean \pm standard deviations.

TUG: timed up and go test, FRTA: functional reach test anterior, FRTP: functional reach test posterior, FES: Falls efficacy scale, STS: sit to stand, 10MWT: 10-m walk test, MBI: modified Barthel index, QoL: quality of life. 


\section{Discussion}

Parkinson's disease occurs because of dopamine deficiency in the cerebral basal ganglia. Patients with Parkinson's disease have a disability in the integration of sensory and movement [30]. For these patients, physical activities and exercises exert a positive effect on the central nervous system. This promotes the recovery of the nervous system and limits the progression of the disease [31].

The purpose of this study was to develop a home exercises program for patients with Parkinson's disease who have difficulty in carrying out ADL and in receiving regular treatment at medical institutions because of serious deterioration of balance, cognitive impairment, depression, and other complications. Home exercises, to date, have been difficult to systematically manage because of low rates of exercise performance and incorrect performance of exercises. Thus, the present study attempted to create a table for patients to self-check themselves and overcome any shortcomings by educating patients and caregivers on a regular basis. Exercise programs that improve balance, walking, and quality of life in patients with Parkinson's disease have been generally reported to be effective [11]. However, it is difficult to substantially apply the exercise programs since patients with Parkinson's disease have different home environments, distances from the hospital, and therapeutic environment [32]. In the present study, which overcame these shortcomings, self-check and regular training were conducted for participants. Thus, they were educated on various items of the home exercises program, such as sitting and standing up, wall push-ups, lying down and lifting the leg, muscle strengthening of the lower extremity including lying down and lifting the buttock, and stretching of the neck, shoulders, and back. The results found a significant difference in quality of life $(\mathrm{p}<.05)$, balance, lower-extremity muscle strength, walking, falls efficacy, and daily life behaviors in patients with Parkinson's disease.

Previous studies have been conducted that reported that home exercises have a positive effect on physical functions in patients with Parkinson's disease; however, research on the method for improving the performance rate of home exercises has been incomplete. Therefore, methods for increasing the performance rate of the home exercises were referred to. Schoo (2005) conducted an experiment involving 115 elderly people with knee and hip osteoarthritis, The results revealed better outcomes in the group who had feedback materials through booklets containing pictures, audio, and video [33]. In addition, another experiment of caregivers of adolescent and pediatric patients with brachial plexus palsy was performed by providing them with DVDs of home exercises. The results revealed functional recovery of the upper extremity, the gaining of confidence, and an increase in the frequency of exercises [34]. Therefore, the present study attempted to increase the performance rate of home exercises by educating not only subjects, but caregivers, issuing brochures of the home-exercises methods, and regularly inspecting a selfmonitored checklist. This method produced positive results.

Studies on the elderly have demonstrated that stretching and muscle-strengthening exercises have a positive effect on the functional mobility of the joints, balance, walking, fall prevention, and enhancement of the quality of life [35-37]. In addition, musclestrengthening exercises for patients with mild or moderate Parkinson's disease caused the improvement of motor functions [38, 39]. Hirsch et al. (2003) reported that high-intensity resistance training and balance training in parallel improved muscular strength and balance abilities [40]. Gobbi et al. (2009) reported that exercise programs in various areas positively impacted walking, balance, and muscular strength, rather than a single exercise program, and thus, had an effect on fall scores, ADL indices, and quality of life [41]. The present study revealed significant results in walking, balance, lower-extremity muscle strength, sensitivity of falls, ADL index, and quality of life by providing an exercises program that was regularly performed, once a day, through four ways of muscle- 
strengthening exercises for the lower and upper extremities and the trunk and via the stretching of all the joints.

The present study evaluated the provision of simple home exercises to patients with Parkinson's disease and continuous feedback for motivation. Although the present study was a cross-sectional study of few subjects, all evaluated indices showed significant values. Thus, the home exercises program of the present study is expected to help patients with Parkinson's disease.

\section{Acknowledgements}

This study was supported by sahmyook university

\section{References}

[1] J. M. Hausdorff, "Rhythmic auditory stimulation modulates gait variability in Parkinson's disease", Eur Journal Neurosci, vol. 26, no. 8, (2007), pp. 2369-2375.

[2] L. E. Dibble, "Sensory cueing effects on maximal speed gait initiation in persons with Parkinson's disease and healthy elders", Gait Posture, vol. 19, no. 3, (2004), pp. 215-225.

[3] S. Papapetropoulos, A. A. Argyriou and J. Ellul, "Factors associated with drug-induced visual hallucinations in Parkinson's disease", Journal Neurol, vol. 252, no. 10, (2005), pp. 1223-1228.

[4] P. Andrell, "Fatality, morbidity and quality of life in patients with refractory angina pectoris", Int $\mathbf{J}$ Cardiol, vol. 147, no. 3, (2011), pp. 377-382.

[5] L. F. Lin, "GDNF: a glial cell line-derived neurotrophic factor for midbrain dopaminergic neurons", Science, vol. 260, no. 5111, (1993), pp. 1130-1132.

[6] R. W. Oppenheim, "Developing motor neurons rescued from programmed and axotomy-induced cell death by GDNF", Nature, vol. 373, no. 6512, (1995), pp. 344-346.

[7] B. Yan, D. Yang and A. Parkinson, "Cloning and expression of hydrolase C, a member of the rat carboxylesterase family", Arch Biochem Biophys, vol. 317, no. 1, (1995), pp. 222-234.

[8] M. E. Morris, "Falls and mobility in Parkinson's disease: protocol for a randomised controlled clinical trial", BMC Neurol, vol. 11, (2011), pp. 93.

[9] I. Reuter, "Effects of a flexibility and relaxation programme, walking, and nordic walking on Parkinson's disease", J Aging Res, vol. 2011, (2011), pp. 232473.

[10] A. D. Speelman, "How might physical activity benefit patients with Parkinson disease?", Nat Rev Neurol, vol. 7, no. 9, (2011), pp. 528-534.

[11] V. A. Goodwin, "The effectiveness of exercise interventions for people with Parkinson's disease: a systematic review and meta-analysis", Mov Disord, vol. 23, no. 5, (2008), pp. 631-640.

[12] L. A. King, "Effects of group, individual, and home exercise in persons with Parkinson disease: a randomized clinical trial", Journal of Neurologic Physical Therapy, vol. 39, no. 4, (2015), pp. 204-212.

[13] J. Nocera, M. Horvat and C.T. Ray, "Effects of home-based exercise on postural control and sensory organization in individuals with Parkinson disease", Parkinsonism \& related disorders, vol. 15, no. 10, (2009), pp. 742-745.

[14] I. Pretzer-Aboff, E. Galik and B. Resnick, "Feasibility and impact of a function focused care intervention for Parkinson's disease in the community", Nursing research, vol. 60, no. 4, (2011), pp. 276-283.

[15] E. M. Simek, "What are the characteristics of home exercise programs that older adults prefer?: a crosssectional study", American Journal of Physical Medicine \& Rehabilitation, vol. 94, no. 7, (2015), pp. 508-521.

[16] "Guidelines for Physiotherapy Practice in Parkinson's Disease", Health Information \& Libraries Journal, vol. 18, no. 4, (2001), pp. 15.

[17] R. W. Bohannon, A.W. Andrews and M.W. Thomas, "Walking speed: reference values and correlates for older adults", J Orthop Sports Phys Ther, vol. 24, no. 2, (1996), pp. 86-90.

[18] R. W. Bohannon, "Comfortable and maximum walking speed of adults aged 20-79 years: reference values and determinants", Age Ageing, vol. 26, no. 1, (1997), pp. 15-19.

[19] S. L. Wolf, "Establishing the reliability and validity of measurements of walking time using the Emory Functional Ambulation Profile", Phys Ther, vol. 79, no. 12, (1999), pp. 1122-1133.

[20] K. Saremi, "Reliability and validity of bilateral thigh and foot accelerometry measures of walking in healthy and hemiparetic subjects", Neurorehabil Neural Repair, vol. 20, no. 2, (2006), pp. 297-305.

[21] C. Zampieri, "The instrumented timed up and go test: potential outcome measure for disease modifying therapies in Parkinson's disease", Journal Neurol Neurosurg Psychiatry, vol. 81, no. 2, (2010), pp. 171176.

[22] P. W. Duncan, "Functional reach: a new clinical measure of balance", J Gerontol, vol. 45, no. 6, (1990), pp. M192-197. 
[23] B. S. Norris and A. Medley, "The effect of balance confidence and context on functional reach ability in healthy older adults", PM R, vol. 3, no. 9, (2011), pp. 811-816.

[24] M. E. Tinetti, D. Richman and L. Powell, "Falls efficacy as a measure of fear of falling", J Gerontol, vol. 45, no. 6, (1990), pp. 239-243.

[25] G. I. Kempen, "Cross-cultural validation of the Falls Efficacy Scale International (FES-I) in older people: results from Germany, the Netherlands and the UK were satisfactory", Disabil Rehabil, vol. 29, no. 2, (2007), pp. 155-162.

[26] S. C. Loewen and B. A. Anderson, "Reliability of the Modified Motor Assessment Scale and the Barthel Index", Phys Ther, vol. 68, no. 7, (1988), pp. 1077-1081.

[27] K. Ghandehari, "Comparative interrater reliability of Asian Stroke Disability Scale, modified Rankin Scale and Barthel Index in patients with brain infarction", ARYA Atheroscler, vol. 8, no. 3, (2012), pp. 153-157.

[28] C. Jenkinson, "Self-reported functioning and well-being in patients with Parkinson's disease: comparison of the short-form health survey (SF-36) and the Parkinson's Disease Questionnaire (PDQ-39)", Age Ageing, vol. 24, no. 6, (1995), pp. 505-509.

[29] L. C. Tan, "Validity and reliability of the PDQ-39 and the PDQ-8 in English-speaking Parkinson's disease patients in Singapore", Parkinsonism Relat Disord, vol. 10, no. 8, (2004), pp. 493-499.

[30] R. Marchese, M. Bove and G. Abbruzzese, "Effect of cognitive and motor tasks on postural stability in Parkinson's disease: a posturographic study", Mov Disord, vol. 18, no. 6, (2003), pp. 652-658.

[31] S. Janezic, "Deficits in dopaminergic transmission precede neuron loss and dysfunction in a new Parkinson model", Proc Natl Acad Sci U S A, vol. 110, no. 42, (2013), pp. E4016-4025.

[32] A. D. Speelman, "How might physical activity benefit patients with Parkinson disease?", Nature Reviews Neurology, vol. 7, no. 9, (2011), pp. 528-534.

[33] A. M. M. Schoo, M. Morris and Q. Bui, "The effects of mode of exercise instruction on compliance with a home exercise program in older adults with osteoarthritis", Physiotherapy, vol. 91, no. 2, (2005), pp. 79-86.

[34] K. M. Murphy, "An assessment of the compliance and utility of a home exercise DVD for caregivers of children and adolescents with brachial plexus palsy: a pilot study", PM R, vol. 4, no. 3, (2012), pp. 190197.

[35] M. A. Fiatarone, "High-intensity strength training in nonagenarians. Effects on skeletal muscle", JAMA, vol. 263, no. 22, (1990), pp. 3029-3034.

[36] J. O. Judge, R. H. Whipple and L. I. Wolfson, "Effects of resistive and balance exercises on isokinetic strength in older persons", J Am Geriatr Soc, vol. 42, no. 9, (1994), pp. 937-946.

[37] L. Wolfson, "Balance and strength training in older adults: intervention gains and Tai Chi maintenance", J Am Geriatr Soc, vol. 44, no. 5, (1996), pp. 498-506.

[38] I. Reuter, "Therapeutic value of exercise training in Parkinson's disease”, Med Sci Sports Exerc, vol. 31, no. 11 , (1999), pp. 1544-1549.

[39] T. A. Scandalis, "Resistance training and gait function in patients with Parkinson's disease", Am J Phys Med Rehabil, vol. 80, no. 1, (2001), pp. 38-43; quiz 44-36.

[40] M. A. Hirsch, "The effects of balance training and high-intensity resistance training on persons with idiopathic Parkinson's disease", Arch Phys Med Rehabil, vol. 84, no. 8, (2003), pp. 1109-1117.

[41] L. T. Gobbi, "Exercise programs improve mobility and balance in people with Parkinson's disease", Parkinsonism Relat Disord, vol. 15 Suppl 3, (2009), pp. S49-52. 
International Journal of Bio-Science and Bio-Technology Vol.8, No.6 (2016) 